\title{
Rare-earth chalcogenides: A large family of triangular lattice spin liquid candidates
}

\author{
Weiwei Liu ${ }^{1,2},{ }^{*}$ Zheng Zhang ${ }^{1,2}, *$ Jianting $\mathrm{Ji}^{1},{ }^{*}$ Yixuan $\mathrm{Liu}^{2}$, Jianshu $\mathrm{Li}^{1,2}$, \\ Xiaoqun Wang ${ }^{3}$, Hechang $\mathrm{Lei}^{2}{ }^{\dagger}{ }^{\dagger}$ Gang $\mathrm{Chen}^{4}{ }^{\ddagger}$ and Qingming Zhang ${ }^{1,5 \S}$ \\ ${ }^{1}$ National Laboratory for Condensed Matter Physics and Institute of Physics, \\ Chinese Academy of Sciences, Beijing 100190, China \\ ${ }^{2}$ Department of Physics and Beijing Key Laboratory of Opto-electronic Functional Materials $\& 3$ Micro-nano Devices, \\ Renmin University of China, Beijing 100872, China \\ ${ }^{3}$ Department of Physics and Astronomy, Shanghai Jiao Tong University, Shanghai 200240, China \\ ${ }^{4}$ State Key Laboratory of Surface Physics and Department of Physics, Fudan University, Shanghai 200433, China and \\ ${ }^{5}$ School of Physical Science and Technology, Lanzhou University, Lanzhou 730000, China
}

(Dated: October 2, 2018)

\begin{abstract}
Frustrated quantum magnets are expected to host many exotic quantum spin states like quantum spin liquid (QSL), and have attracted numerous interest in modern condensed matter physics. The discovery of the triangular lattice spin liquid candidate $\mathrm{YbMgGaO}_{4}$ stimulated an increasing attention on the rare-earth-based frustrated magnets with strong spin-orbit coupling. Here we report the synthesis and characterization of a large family of rare-earth chalcogenides $\mathrm{AReCh}_{2}(\mathrm{~A}=$ alkali or monovalent ions, $\mathrm{Re}=$ rare earth, $\mathrm{Ch}=\mathrm{O}, \mathrm{S}, \mathrm{Se})$. The family compounds share the same structure $(\mathrm{R} \overline{3} \mathrm{~m})$ as $\mathrm{YbMgGaO}_{4}$, and antiferromagnetically coupled rare-earth ions form perfect triangular layers that are well separated along the $c$-axis. Specific heat and magnetic susceptibility measurements on $\mathrm{NaYbO}_{2}, \mathrm{NaYbS}_{2}$ and $\mathrm{NaYbSe}_{2}$ single crystals and polycrystals, reveal no structural or magnetic transition down to $50 \mathrm{mK}$. The family, having the simplest structure and chemical formula among the known QSL candidates, removes the issue on possible exchange disorders in $\mathrm{YbMgGaO}_{4}$. More excitingly, the rich diversity of the family members allows tunable charge gaps, variable exchange coupling, and many other advantages. This makes the family an ideal platform for fundamental research of QSLs and its promising applications.
\end{abstract}

PACS numbers: 75.10.Kt, 75.30.Et, 75.30.Gw

Introduction.-The concept of quantum spin liquids (QSLs) was originally proposed by P. W. Anderson theoretically over 40 years ago [1]. It describes a highly entangled quantum state for spin degrees of freedom and was initially constructed with a superposition of spin singlets on the triangular antiferromagnet, so-called resonatingvalence-bond state [1]. Later on, the possible connection between QSLs and high-temperature superconductivity was theoretically established through doping a QSL Mott insulator [2]. Although the underlying mechanism for the high-temperature superconductivity has not yet come into a consensus, our understanding of QSLs has greatly improved, both from exactly solvable models [3, 4] and several classification schemes $[4,5]$. On the experimental side, various frustrated magnetic materials, particularly the triangular-lattice-based antiferromagnets, were considered to be the most promising systems to realize QSLs [6]. So far, a number of compounds have been reported to host QSLs. Among them, the well-known ones include herbertsmithite and its derived compounds [714], and triangular organics [15-19]. The magnetic ions in most of these compounds are $3 d$ transition metal ions $\mathrm{Cu}^{2+}$ with $S=1 / 2$, which may be crucial to enhance quantum fluctuations.

Quite recently, frustrated materials with magnetic rare-earth ions are proposed to be promising QSL candidates [20]. These include the well-known pyrochlore ice materials [21-30], the kagome magnet [31, 32], and the triangular lattice magnets [33-47]. The local degree of freedom for the rare-earth ions that contain an odd number of $4 f$ electrons (excluding $\mathrm{Gd}^{3+}$ ), is a Kramers doublet and can be mapped to an effective spin $S=1 / 2$ degree of freedom. This effective-spin local moment is protected by time reversal symmetry and the point group symmetry. In many cases the non-Kramers rare-earth ions can be taken as effective spin $S=1 / 2$ local moments at low temperatures, though lacking the protection from time reversal symmetry [26, 30, 47-49]. The spin-orbitentangled nature of the rare-earth local moments often brings highly anisotropic spin models that have never been constructed and studied before [26, 45-49]. Thus, the rare-earth-based magnets play an important role in the exploration of novel spin models and the exotic magnetic states on various lattices. Indeed, QSL behaviors and multipolar phases have been proposed for various rare-earth compounds [21-30, 32, 46, 47].

The recent discovery of the triangular lattice magnet $\mathrm{YbMgGaO}_{4}$ has invoked a further interest in the search of spin liquids with strong spin-orbit coupling [33, 34, $44,45,50-54]$. The compound has a space group symmetry of $\mathrm{R} \overline{3} \mathrm{~m}$, and the $\mathrm{Yb}^{3+}$ ions form a flat and perfect triangular lattice $[33,34]$ The availability of highquality single crystals allows extensive and careful studies of magnetic properties using neutron scattering [36-39], muon spin relaxation (muSR) [35], electron spin resonance (ESR) [34] etc. These studies point to a possible gapless U(1) QSL ground state [33-37, 39, 40, 52, 53]. On the other hand, some experiments and theoretical 


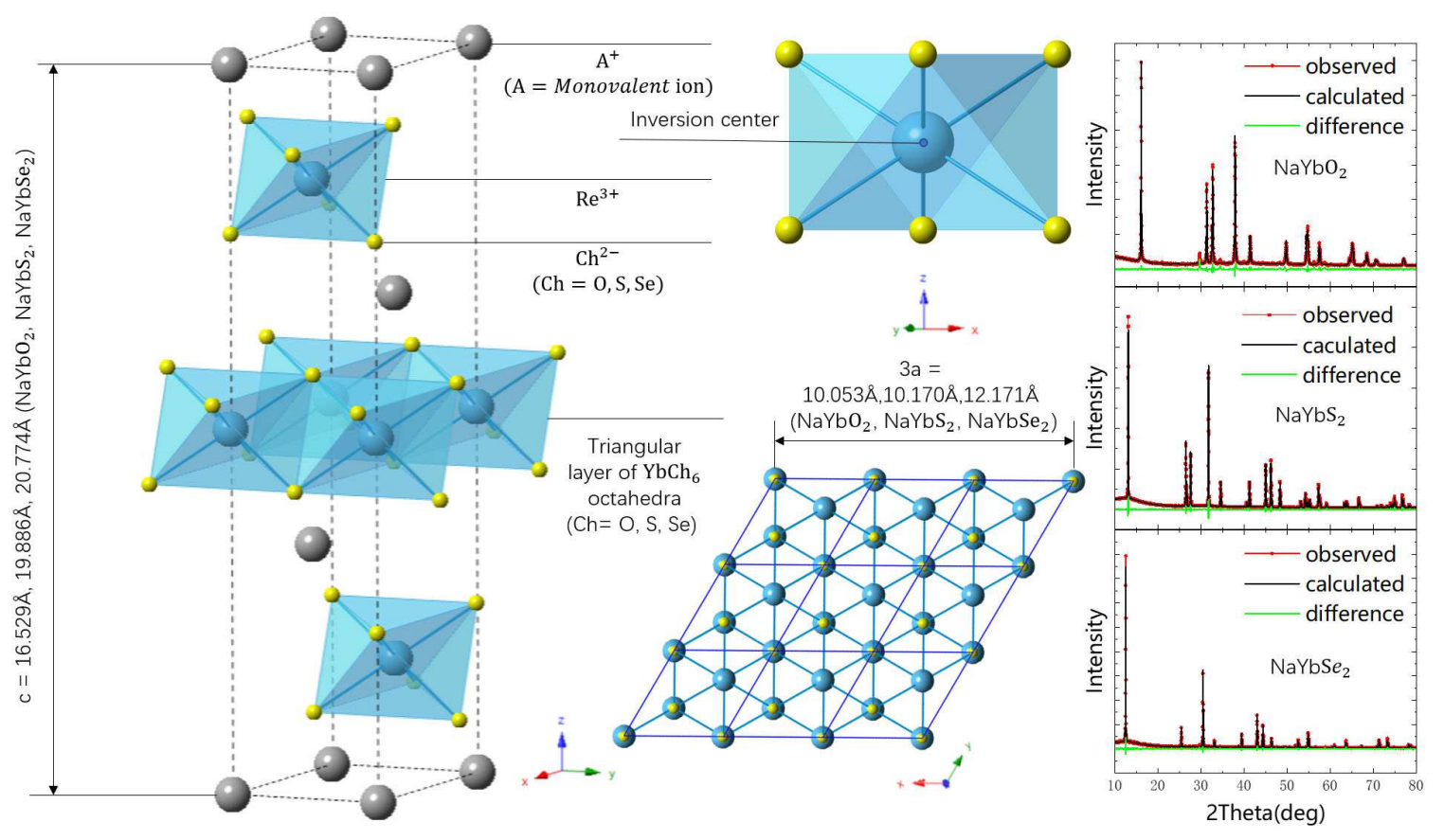

FIG. 1. (Color online.) The general crystal structure of rare-earth chalcogenides and the powder diffraction patterns and Rietveld refinements for $\mathrm{NaYbCh}_{2}(\mathrm{Ch}=\mathrm{O}, \mathrm{S}, \mathrm{Se})$.

arguments raised the issue on $\mathrm{Ga} / \mathrm{Mg}$ disorder, which was suggested to be responsible for the disordered spin state and/or QSL stability [33, 37, 39, 54-56]. The small exchange coupling allows an easy tunability of the spin state with a laboratory magnetic field [33, 37, 39, 44]. Meanwhile, it also requires that most experiments must be carried out at ultralow temperatures. In some cases this could be an obstacle for in-depth studies and possible applications.

As mentioned above, there is a long list of rare-earth magnets $[20,45,47]$. Then the question is if one can find out some interesting compounds or systems with larger exchange couplings and without disorder. This is the purpose of this work. We systematically synthesized the rare-earth chalcogenides $\mathrm{AReCh}_{2}(\mathrm{~A}=$ alkali or monovalent ions, $\mathrm{Re}=$ rare earth, $\mathrm{Ch}=\mathrm{O}, \mathrm{S}, \mathrm{Se}$ ) with a delafossite structure. We carried out the structural and thermodynamic characterizations of these compounds. The compounds have a high symmetry of R $\overline{3} \mathrm{~m}$ and perfect spin triangular layers. The magnetic measurements indicate that spins are antiferromagnetically coupled in all the compounds with a range of Curie-Weiss temperatures. For the representative $\mathrm{NaYbCh}_{2}(\mathrm{Ch}=$ $\mathrm{O}, \mathrm{S}, \mathrm{Se}$ ) samples, no magnetic ordering or transition is observed in the specific heat and susceptibility measurements down to $50 \mathrm{mK}$. Thus, this is a large family of QSL candidates with the simplest structure and chemical for- mula so far. Its crystal structure naturally removes the issue on $\mathrm{Ga} / \mathrm{Mg}$ disorder proposed for $\mathrm{YbMgGaO}_{4}$. The diversity of the large family makes it an ideal playground for studying the QSL physics and exploring its promising applications.

Sample preparation and experimental methods.-The polycrystals of $\mathrm{NaReO}_{2}(\mathrm{Re}=\mathrm{Yb}, \mathrm{Lu})$ were synthesized by the method of solid-state reaction under high temperatures:

$$
\mathrm{Na}_{2} \mathrm{CO}_{3}+\mathrm{Re}_{2} \mathrm{O}_{3} \rightarrow 2 \mathrm{NaReO}_{2}+\mathrm{O}_{2} \quad(\mathrm{Re}=\mathrm{Yb}, \mathrm{Lu}) .
$$

$\mathrm{Na}_{2} \mathrm{CO}_{3}$ and $\mathrm{Re}_{2} \mathrm{O}_{3}$ powders were mixed in a dry process (mixing molar ratio: $\mathrm{Na}_{2} \mathrm{O}: \mathrm{Yb}_{2} \mathrm{O}_{3}=2.5: 1$ ) and shaped into a pellet by isostatic pressing (50MPa, $2 \mathrm{~min})$. Shaped samples were heated at 900 degrees for 9 hours. After the heating, the samples were ground and washed with distilled water and ethanol, at lastly dried in air at room temperature for 48 hours. The polycrystals of $\mathrm{NaReS}_{2}$ $(\mathrm{Re}=\mathrm{La}, \mathrm{Ce}, \mathrm{Pr}, \mathrm{Nd}, \mathrm{Sm}, \mathrm{Eu}, \mathrm{Gd}, \mathrm{Tb}, \mathrm{Dy}, \mathrm{Ho}, \mathrm{Er}, \mathrm{Tm}$, $\mathrm{Yb}, \mathrm{Lu}$ ) were synthesized by the method of solid-state reaction under high temperature:

$$
\mathrm{Na}_{2} \mathrm{~S}+2 \mathrm{Re}+3 \mathrm{~S} \rightarrow 2 \mathrm{NaReS}_{2},
$$

where Re = Lu, Se, Tm, Er, Ho, Dy, Tb, Gd, Eu, Sm, Nd, $\mathrm{Pr}, \mathrm{Ce}, \mathrm{La}$. The $\mathrm{Na}_{2} \mathrm{~S}$, Re and $\mathrm{S}$ powder was mixed in $\mathrm{Ar}$ environment glove box. The mixed powders were placed in a graphite crucible and vacuum packaged with quartz 


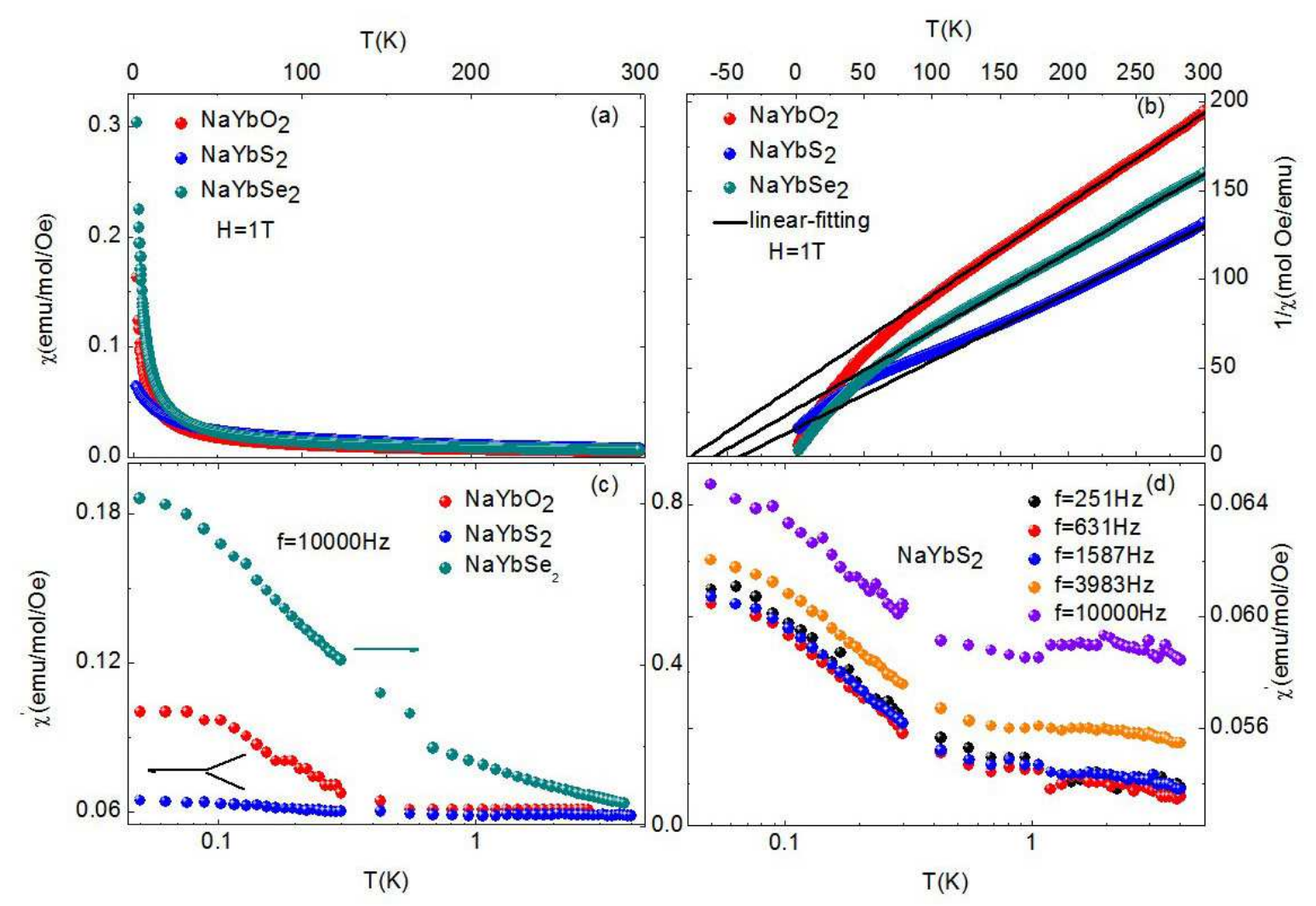

FIG. 2. (Color online.) The DC (a \& b) and AC (c \& d) magnetic susceptibilities of polycrystalline $\mathrm{NaYbCh}_{2}(\mathrm{Ch}=\mathrm{O}, \mathrm{S}, \mathrm{Se})$.

tube. Packaged samples were heated at 850 degrees for 48 hours. After the heating, the sample were powder and washed with distilled water and then dried in air at 50 degrees for 6 hours. The synthesis of polycrystals of $\mathrm{NaReSe}_{2}(\mathrm{Re}=\mathrm{Er}, \mathrm{Yb}, \mathrm{Lu})$ were similar with the synthesis of powders of $\mathrm{NaReS}_{2}$. The temperature was adjusted to 900 degrees.

We have also successfully grown high quality $\mathrm{NaYbSe}_{2}$ single crystals. The growth conditions of single crystals are more rigorous than $\mathrm{NaYbSe}_{2}$ polycrystals. $\mathrm{Na}_{2} \mathrm{Se}$, $\mathrm{Yb}$ and Se powders were mixed in Ar environment glove box (mixing molar ratio: $\mathrm{Na}_{2} \mathrm{Se}: \mathrm{Yb}: \mathrm{Se}=1: 2: 12$ ). The mixed powder was placed in special quartz tube that can withstand higher pressure. Packaged samples were headed at 1000 degrees for 48 hours. After the heating, we can observe $2-3 \mathrm{~mm}$ size single crystals. We made a simple resistance check for the crystals and the resistance is overranged and thus $\mathrm{NaYbSe}_{2}$ is confirmed to be a good insulator.

Powder XRD profiles were measured with Bruker-D8 by step scanning. The TOPAS program was used for Rietveld crystal structure refinements. The temperature dependence of magnetic susceptibility from $1.8 \mathrm{~K}$ to $300 \mathrm{~K}$ was measured with a SQUID magnetometer (Quantum Design Magnetic Property Measurement System, MPMS) under both ZFC and FC for all the samples with

\begin{tabular}{lcccc}
\hline \hline Ch Space group & $\mathrm{C}$ & \multicolumn{2}{c}{$\Theta_{\mathrm{CW}} / \mathrm{K}$} & $\mu_{\mathrm{eff}}\left(\mu_{\mathrm{B}}\right)$ \\
\hline \hline $\mathrm{O}$ & $\mathrm{R} 3 \mathrm{~m}$ & 1.9448 & -77.35 & 3.94 \\
$\mathrm{~S}$ & $\mathrm{R} \overline{3} \mathrm{~m}$ & 2.6166 & -40.98 & 4.57 \\
$\mathrm{Se}$ & $\mathrm{R} \overline{3} \mathrm{~m}$ & 2.2550 & -59.84 & 4.24 \\
\hline \hline
\end{tabular}

TABLE I. Parameters extracted from Curie-Weiss fitting for $\mathrm{NaYbCh}_{2}(\mathrm{Ch}=\mathrm{O}, \mathrm{S}, \mathrm{Se})$.

the brass sample holder. The AC susceptibility measurements from $50 \mathrm{mK}$ to $4 \mathrm{~K}$ were performed using a dilution refrigeration system (DR). The polycrystalline sample was pressed into a thin plate and fixed on a sample holder with GE vanish. The heat capacity measurements from $2 \mathrm{~K}$ to $30 \mathrm{~K}$ were performed using PPMS (Quantum Design Physical Property Measurement System) and DR was employed for the measurements from $50 \mathrm{mK}$ to $4 \mathrm{~K}$. The plate sample was mounted on a sample holder with $\mathrm{N}$ grease for a better thermal contact.

Results and discussions. - In Fig. 1, we depict the crystal structure of the rare-earth chalcogenides and the Rietveld refinements for three representative samples $\mathrm{NaYbO}_{2}, \mathrm{NaYbS}_{2}$ and $\mathrm{NaYbSe}_{2}$, where the detailed structural information extracted from the refinements can be found in the Supplementary Materials. The system has an $R \overline{3} \mathrm{~m}$ space group symmetry, and 


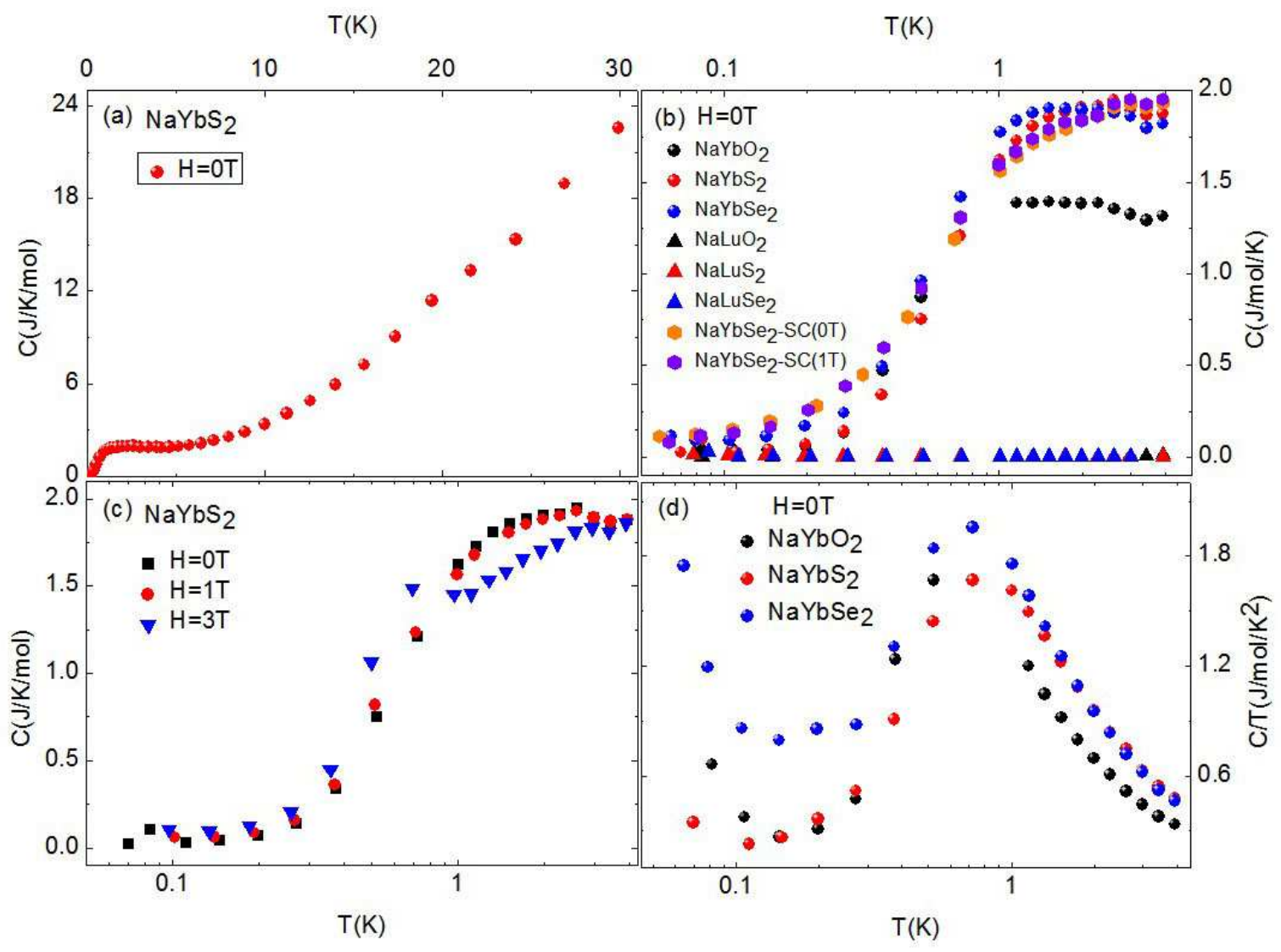

FIG. 3. (Color online.) Specific heat measurements on $\mathrm{NaYbCh}_{2}(\mathrm{Ch}=\mathrm{O}, \mathrm{S}, \mathrm{Se})$. Single crystal (SC) $\mathrm{NaYhSe}_{2}$ and polycrystalline $\mathrm{NaYbO}_{2}$ and $\mathrm{NaYbS}_{2}$ were used in the measurements.

the magnetic ions form flat triangular layers that are well separated. The $\mathrm{ReCh}_{6}$ octahedra are connected in an edge-sharing fashion. The local crystal-field environment around the magnetic ions is exactly analogous to the case of $\mathrm{YbMgGaO}_{4}$. Therefore, one expects a similar crystal-field splitting scheme of the $\mathrm{Yb}^{3+}$ ions as the one in $\mathrm{YbMgGaO}_{4}$. This means that a spin-orbitalentangled effective spin $S=1 / 2$ local moment should hold for our case. Similar to $\mathrm{YbMgGaO}_{4}$, the antisymmetric Dzyaloshinskii-Moriya interaction is prohibited by the inversion symmetry of the system.

The $\mathrm{Mg} / \mathrm{Ga}$ disorder in the non-magnetic layers of $\mathrm{YbMgGaO} 4$ has been extensively discussed and is still under debate. Whether or how much this non-magnetic disorder impacts on the $\mathrm{Yb}$ magnetic properties is unclear in this stage. In some experiments and theoretical calculations, the disorder was considered to play a dominant role in contributing to the low-energy excitations. As a comparison, there is no such disorder in this family of rare-earth chalcogenides, due to the structural simplicity. The issue on disorder is completely removed for this family of materials. We further made the analysis of element ratio (See Supplementary Materials), which is close to the nominal ratio. This rules out the possibility of the disorder caused by element deficiency. If one still concerns about the active monovalent ions like $\mathrm{Na}^{+}$and $\mathrm{K}^{+}$, he will have plenty of choices of the heavy monovalent ones such as $\mathrm{Rb}^{+}, \mathrm{Cs}^{+}, \mathrm{Cu}^{+}$and $\mathrm{Ag}^{+}$, etc.

For the selected sub-family $\mathrm{NaYbCh}_{2}(\mathrm{Ch}=\mathrm{O}, \mathrm{S}, \mathrm{Se})$, we measured the DC magnetic susceptibility in the range of $2-300 \mathrm{~K}$ and the AC susceptibility from $50 \mathrm{mK}$ to $4 \mathrm{~K}$. The results are presented in Fig. 2. The Curie-Weiss fitting was made from $150 \mathrm{~K}$ to $300 \mathrm{~K}$ according to the crystal-field splitting in $\mathrm{YbMgGaO}_{4}$ and the fitting results are summarized in Table. I. Considering the small interaction energy scale of rare-earth moments, this fitting range may not be quite sufficient to characterize the low-energy magnetic physics of the system. A lower fitting range may be required in the future. Nevertheless, the negative Curie-Weiss temperatures suggest an antiferromagnetic coupling in all the samples. Excitingly, the Curie-Weiss temperatures are much larger than that of $\mathrm{YbMgGaO}_{4}$ because of the smaller distances between nearest-neighbor $\mathrm{Yb}^{3+}$ in the rare-earth chalcogenides. The AC susceptibility for the three samples shows no sign of long-range magnetic ordering. The measurements under various frequencies further confirms that there is no spin freezing either. Interestingly, the susceptibility sat- 


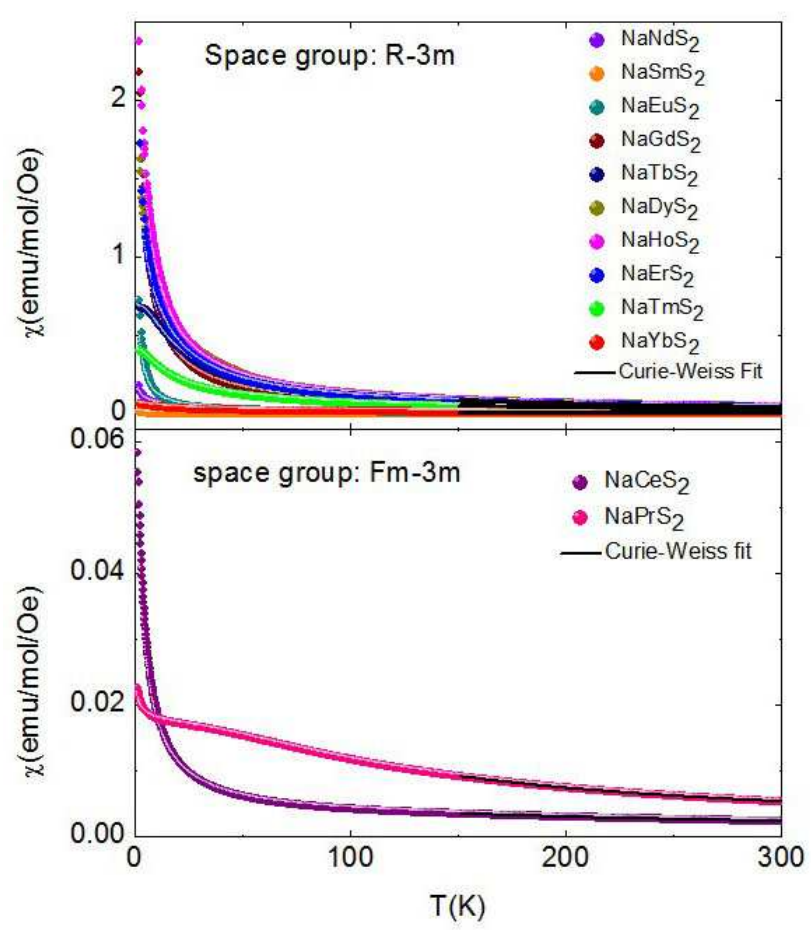

FIG. 4. (Color online.) Magnetic susceptibility of $\mathrm{NaReS}_{2}$ $(\mathrm{Re}=\mathrm{Ce}-\mathrm{Yb})$ in the range of $2 \mathrm{~K}$ to $300 \mathrm{~K}$.

uration in the zero temperature limit is clearly observed for all the three samples. This should be one of the consequences caused by strong spin-orbit coupling rather than a sign of finite density of spin excitations. Under the strong spin-orbit coupling, the total magnetization is not a good quantum number and cannot be used to label the many-body eigenstate. The many-body eigenstate would be a mixture of states with different total magnetizations. The magnetic susceptibility would always be a constant. We also find that the low-temperature susceptibility of $\mathrm{NaYbSe}_{2}$ is obviously larger than that of the other two compounds. In fact, the distance between nearest neighbor $\mathrm{Yb}^{3+}$ ions in $\mathrm{NaYbSe}_{2}$ is larger, while its Curie-Weiss temperatures and the moment obtained from the Curie-Weiss fitting look comparable to the other two.

Our specific heat results are shown in Fig. 3. There is no obvious transition down to $50 \mathrm{mK}$ in these compounds, and it is consistent with the conclusion from the magnetic susceptibility data. We observed no apparent change or shift with applying a magnetic field up to $3 \mathrm{~T}$ (see Fig. 3c). On the other hand, an upturn is observed below $100 \mathrm{mK}$ in the $C / T-T$ plot (see Fig. $3 \mathrm{~d}$ ). This may arise from the nuclear Schottky anomaly due to the nuclear spins. The upturn makes it difficult to obtain the intrinsic trend of the specific heat below $100 \mathrm{mK}$ and to conclude whether the system is a gapless or gapped QSL. Thus, more detailed magnetic and thermodynamic exper- iments are required in the future. The broad peak around $1 \mathrm{~K}$ in the $C / T-T$ plot is considered to be a consequence of preserving entropy. Here we point out that there is no obvious disorder in the present case and we still do not observe any long-range magnetic ordering or freezing that points to a possible QSL ground state. This means that the rare-earth triangular system, including rare-earth chalcogenides reported here and $\mathrm{YbMgGaO}_{4}$, intrinsically hosts the QSL state that is not stabilized by or even originated from the $\mathrm{Ga} / \mathrm{Mg}$ charge disorder.

The measurements discussed above are based on the sub-family $\mathrm{NaYbCh}_{2}(\mathrm{Ch}=\mathrm{O}, \mathrm{S}, \mathrm{Se})$. Towards a comprehensive view of the large family, we fixed $\mathrm{Na}$ and S, and systematically synthesized the other sub-family $\mathrm{NaReS}_{2}(\mathrm{Re}=\mathrm{La}-\mathrm{Lu})$. The Rietveld refinements for all the fourteen compounds have been made and the detailed structural parameters can be found in the Supplementary Materials. The family members from Nd to $\mathrm{Lu}$ preserve the high lattice symmetry of $\mathrm{R} \overline{3} \mathrm{~m}$. But the three end members, $\mathrm{NaLaS}_{2}, \mathrm{NaCeS}_{2}$ and $\mathrm{NaPrS}_{2}$ show a cubic structure with the space group symmetry of $\mathrm{Fm} \overline{3} \mathrm{~m}$. Clearly this is caused by the larger ionic radii of La, Ce and Pr. The magnetic susceptibility measurements from $2 \mathrm{~K}$ to $300 \mathrm{~K}$ have been carried out on the sub-family and the results are presented in Fig. 4. No obvious magnetic transition is observed in all the compounds with the $\mathrm{R} \overline{3} \mathrm{~m}$ symmetry. Similar to the $\mathrm{R} \overline{3} \mathrm{~m}$ brothers, the cubic $\mathrm{NaCeS}_{2}$ also shows no sign of magnetic transition from $2 \mathrm{~K}$ to $300 \mathrm{~K}$, as the perfect triangular lattice formed by the magnetic ions remains undistorted and the strong geometrical frustration is always there. In contrast, an anomaly appears around $10 \mathrm{~K}$ in the susceptibility of $\mathrm{NaPrS}_{2}(\mathrm{Fm} \overline{3} \mathrm{~m})$.

The Curie-Weiss fitting was performed from $150 \mathrm{~K}$ to $300 \mathrm{~K}$, assuming a reasonably high crystal field splitting. The full results are presented in Table II. We can see that the exchange couplings vary from sample to sample. In the other words, we have the opportunity to select the compounds with various exchange coupling. Beyond this, one can further tune the charge gaps of the family members by element substitution. The absorption spectra (see Supplementary Materials) indicate that the charge gaps are roughly $4.5 \mathrm{eV}, 2.7 \mathrm{eV}$ and $1.9 \mathrm{eV}$ for $\mathrm{NaYbO}_{2}$, $\mathrm{NaYbS}_{2}$, and $\mathrm{NaYbSe}_{2}$, respectively. The variable and small charge gaps may allow the system to access a Mottmetal transition by applying doping or pressures. Such a possibility opens up the interesting direction of Mott transitions out of a QSL [57]. This transition was argued to be continuous by noticing that the Landau damping term scales like a mass term for the bosonic charge and then identifying the transition as an usual superfluidMott transition [57]. Thus, these exciting advantages stem from the rich diversity of the family. In fact, we have made a careful literature research and found that most of the family members have the high-symmetry of $\mathrm{R} \overline{3} \mathrm{~m}$ and hence are potential QSL materials (see Supple- 


\begin{tabular}{lcclll}
\hline \hline Re & Space group & $\mathrm{C}$ & \multicolumn{1}{c}{$\Theta_{\mathrm{CW}} / \mathrm{K}$} & $\mu_{\text {eff }}$ (Obs.) & $\mu_{\text {eff }}$ (Cal.) \\
\hline \hline $\mathrm{Ce}$ & $\mathrm{F} m \overline{3} m$ & 1.1145 & -164.42 & $2.99 \mu_{\mathrm{B}}$ & $2.54 \mu_{\mathrm{B}}$ \\
$\mathrm{Pr}$ & $\mathrm{F} m \overline{3} m$ & 1.91757 & -57.48 & $3.92 \mu_{\mathrm{B}}$ & $3.58 \mu_{\mathrm{B}}$ \\
$\mathrm{Nd}$ & $\mathrm{R} \overline{3} \mathrm{~m}$ & 1.78198 & -25.35 & $3.77 \mu_{\mathrm{B}}$ & $3.62 \mu_{\mathrm{B}}$ \\
$\mathrm{Sm}$ & $\mathrm{R} \overline{3} \mathrm{~m}$ & 0.71761 & -343.05 & $2.40 \mu_{\mathrm{B}}$ & $0.84 \mu_{\mathrm{B}}$ \\
$\mathrm{Eu}$ & $\mathrm{R} \overline{3} \mathrm{~m}$ & 3.19082 & -106.13 & $5.05 \mu_{\mathrm{B}}$ & $3.6 \mu_{\mathrm{B}}$ \\
$\mathrm{Gd}$ & $\mathrm{R} \overline{3} \mathrm{~m}$ & 8.5521 & -1.98 & $8.27 \mu_{\mathrm{B}}$ & $7.94 \mu_{\mathrm{B}}$ \\
$\mathrm{Tb}$ & $\mathrm{R} \overline{3} \mathrm{~m}$ & 12.87953 & -9.49 & $10.15 \mu_{\mathrm{B}}$ & $9.72 \mu_{\mathrm{B}}$ \\
$\mathrm{Dy}$ & $\mathrm{R} \overline{3} \mathrm{~m}$ & 14.55906 & -9.39 & $10.79 \mu_{\mathrm{B}}$ & $10.63 \mu_{\mathrm{B}}$ \\
$\mathrm{Ho}$ & $\mathrm{R} \overline{3} \mathrm{~m}$ & 13.8468 & -5.90 & $10.52 \mu_{\mathrm{B}}$ & $10.60 \mu_{\mathrm{B}}$ \\
$\mathrm{Er}$ & $\mathrm{R} \overline{3} \mathrm{~m}$ & 12.13713 & -4.62 & $9.85 \mu_{\mathrm{B}}$ & $9.59 \mu_{\mathrm{B}}$ \\
$\mathrm{Tm}$ & $\mathrm{R} \overline{3} \mathrm{~m}$ & 7.40589 & -3.83 & $7.69 \mu_{\mathrm{B}}$ & $7.57 \mu_{\mathrm{B}}$ \\
$\mathrm{Yb}$ & $\mathrm{R} \overline{3} \mathrm{~m}$ & 2.90963 & -63.74 & $4.82 \mu_{\mathrm{B}}$ & $4.54 \mu_{\mathrm{B}}$ \\
\hline \hline
\end{tabular}

TABLE II. Parameters extracted from the Curie-Weiss fitting for $\mathrm{NaReS}_{2}(\mathrm{Re}=\mathrm{Ce}-\mathrm{Yb})$.

mentary Materials). This suggests that the family is an ideal playground, on which we can tune the basic material parameters or exchange coupling to explore the QSL physics and develop its possible applications.

Summary.-In summary, we have synthesized rareearth chalcogenides $\mathrm{AReCh}_{2}$ that exhibit a delafossite structure, and made structural and thermodynamics characterizations. The family has a lattice symmetry $\mathrm{R} \overline{3} \mathrm{~m}$, and the magnetic ions are antiferromagnetically coupled and form perfect triangular layers. The magnetic susceptibility and specific heat measurements down to $50 \mathrm{mK}$ indicate no sign of long-range magnetic ordering or transition. The family removes the disorder issue raised in $\mathrm{YbMgGaO}_{4}$ and also suggests the QSL physics is probably not from disorder. The unique advantages, such as various charge gaps and exchange coupling, suggest that the family may be an ideal platform for the further study of QSLs.

Acknowledgments.-We thank Zicheng Wen for assisting the absorption measurements and Feng Jin for organizing the references. This work is supported by the Ministry of Science and Technology of China (2016YFA0300504 \& 2017YFA0302904 \& 2016YFA0301001) and the NSF of China (11774419, 11474357, 11822412, 11774423 \& 11574394).

Note added: Upon the completion of this work, we become aware of Ref. 58 that focused on $\mathrm{NaYbS}_{2}$ and proposed it as a spin liquid candidate.

\footnotetext{
* These authors contributed equally to this work.

$\dagger$ hlei@ruc.edu.cn

‡ chggst@gmail.com

$\S$ qmzhang@iphy.ac.cn

[1] P.W. Anderson, Mater. Res. Bull. 8, 153-60 (1973).

[2] Patrick A. Lee, Naoto Nagaosa, and Xiao-Gang Wen, "Doping a mott insulator: Physics of high-temperature superconductivity," Rev. Mod. Phys. 78, 17-85 (2006).

[3] A. Kitaev, Annals of Physics 321, 2-111 (2006).
}

[4] X.-G. Wen, Quantum Field Theory of Many-body Systems: From the Origin of Sound to an Origin of Light and Electrons, reissue ed. (Oxford University Press, New York, USA, 2007).

[5] Andrew M. Essin and Michael Hermele, "Classifying fractionalization: Symmetry classification of gapped $\digamma_{2}$ spin liquids in two dimensions," Phys. Rev. B 87, 104406 (2013).

[6] Leon Balents, "Spin liquids in frustrated magnets," Nature 464, 199-208 (2010).

[7] J. S. Helton, K. Matan, M. P. Shores, E. A. Nytko, B. M. Bartlett, Y. Yoshida, Y. Takano, A. Suslov, Y. Qiu, J.H. Chung, D. G. Nocera, and Y. S. Lee, "Spin Dynamics of the Spin-1/2 Kagome Lattice Antiferromagnet $\mathrm{ZnCu}_{3}(\mathrm{OH})_{6} \mathrm{Cl}_{2}$," Phys. Rev. Lett. 98, 107204 (2007).

[8] Tian-Heng Han, Joel S Helton, Shaoyan Chu, Daniel G Nocera, Jose A Rodriguez-Rivera, Collin Broholm, and Young S Lee, "Fractionalized excitations in the spinliquid state of a kagome-lattice antiferromagnet," Nature 492, 406-410 (2012).

[9] A. Olariu, P. Mendels, F. Bert, F. Duc, J. C. Trombe, M. A. de Vries, and A. Harrison, " 17 O NMR Study of the Intrinsic Magnetic Susceptibility and Spin Dynamics of the Quantum Kagome Antiferromagnet $\mathrm{ZnCu}_{3}(\mathrm{OH})_{6} \mathrm{Cl}_{2}$," Phys. Rev. Lett. 100, 087202 (2008).

[10] Yoshihiko Okamoto, Hiroyuki Yoshida, and Zenji Hiroi, J. Phys. Soc. Jpn. 78, 033701 (2009).

[11] Zenji Hiroi, Masafumi Hanawa, Naoya Kobayashi, Minoru Nohara, Hidenori Takagi, Yoshitomo Kato, and Masashi Takigawa, "Spin-1/2 KagomLike Lattice in Volborthite $\mathrm{Cu}_{3} \mathrm{~V}_{2} \mathrm{O}_{7}(\mathrm{OH})_{2} \cdot 2 \mathrm{H}_{2} \mathrm{O}$," Journal of the Physical Society of Japan 70, 3377-3384 (2001).

[12] M. Yoshida, M. Takigawa, H. Yoshida, Y. Okamoto, and Z. Hiroi, "Phase diagram and spin dynamics in volborthite with a distorted kagome lattice," Phys. Rev. Lett. 103, 077207 (2009).

[13] Yuesheng Li, Bingying Pan, Shiyan Li, Wei Tong, Langsheng Ling, Zhaorong Yang, Junfeng Wang, Zhongjun Chen, Zhonghua Wu, and Qingming Zhang, "Gapless quantum spin liquid in the $\mathrm{S}=1 / 2$ anisotropic kagome antiferromagnet $\mathrm{ZnCu}_{3}(\mathrm{OH})_{6} \mathrm{SO}_{4}$," New Journal of Physics 16, 093011 (2014).

[14] A. Zorko, S. Nellutla, J. van Tol, L. C. Brunel, F. Bert, F. Duc, J.-C. Trombe, M. A. de Vries, A. Harrison, and P. Mendels, "Dzyaloshinsky-Moriya Anisotropy in the Spin-1/2 Kagome Compound $\mathrm{ZnCu}_{3}(\mathrm{OH})_{6} \mathrm{Cl}_{2}$," Phys. Rev. Lett. 101, 026405 (2008).

[15] T Itou, A Oyamada, S Maegawa, M Tamura, and $\mathrm{R}$ Kato, "Spin-liquid state in an organic spin- $1 / 2$ system on a triangular lattice, EtMe $\left.\mathrm{Eb}_{3} \mathrm{Sbd}(\mathrm{Pmit})_{2}\right]_{2}$," Journal of Physics: Condensed Matter 19, 145247 (2007).

[16] T. Itou, A. Oyamada, S. Maegawa, M. Tamura, and R. Kato, "Quantum spin liquid in the spin12 triangular antiferromagnet $\mathrm{Etme}_{3} \mathrm{Sb}\left[\mathrm{Pd}(\mathrm{dmit})_{2}\right]_{2}$," Phys. Rev. B 77, 104413 (2008).

[17] Y. Shimizu, K. Miyagawa, K. Kanoda, M. Maesato, and G. Saito, "Spin liquid state in an organic mott insulator with a triangular lattice," Phys. Rev. Lett. 91, 107001 (2003).

[18] Satoshi Yamashita, Yasuhiro Nakazawa, Masaharu Oguni, Yugo Oshima, Hiroyuki Nojiri, Yasuhiro Shimizu, Kazuya Miyagawa, and Kazushi Kanoda, "Thermodynamic properties of a spin- $1 / 2$ spin-liquid state in a kappa-type organic salt," 
Nature Physics 4, 459-462 (2008).

[19] Y. Kurosaki, Y. Shimizu, K. Miyagawa, K. Kanoda, and G. Saito, "Mott Transition from a Spin Liquid to a Fermi Liquid in the SpinFrustrated Organic Conductor $\kappa-(\mathrm{ET})_{2} \mathrm{Cu}_{2}(\mathrm{CN})_{3}$," Phys. Rev. Lett. 95, 177001 (2005).

[20] Jason S. Gardner, Michel J. P. Gingras, and John E. Greedan, "Magnetic pyrochlore oxides," Rev. Mod. Phys. 82, 53-107 (2010).

[21] J. S. Gardner, S. R. Dunsiger, B. D. Gaulin, M. J. P. Gingras, J. E. Greedan, R. F. Kiefl, M. D. Lumsden, W. A. MacFarlane, N. P. Raju, J. E. Sonier, I. Swainson, and Z. Tun, "Cooperative Paramagnetism in the Geometrically Frustrated Pyrochlore Antiferromagnet $\mathrm{Tb}_{2} \mathrm{Ti}_{2} \mathrm{O}_{7}$," Phys. Rev. Lett. 82, 1012-1015 (1999).

[22] K. A. Ross, J. P. C. Ruff, C. P. Adams, J. S. Gardner, H. A. Dabkowska, Y. Qiu, J. R. D. Copley, and B. D. Gaulin, "Two-Dimensional Kagome Correlations and Field Induced Order in the Ferromagnetic XY Pyrochlore $\mathrm{Yb}_{2} \mathrm{Ti}_{2} \mathrm{O}_{7}$," Phys. Rev. Lett. 103, 227202 (2009).

[23] A. J. Princep, D. Prabhakaran, A. T. Boothroyd, and D. T. Adroja, "Crystal-field states of $\mathrm{Pr}^{3+}$ in the candidate quantum spin ice $\operatorname{Pr}_{2} \mathrm{Sn}_{2} \mathrm{O}_{7}$," Phys. Rev. B 88, 104421 (2013).

[24] Kate A. Ross, Lucile Savary, Bruce D. Gaulin, and Leon Balents, "Quantum excitations in quantum spin ice," Phys. Rev. X 1, 021002 (2011).

[25] Hamid R. Molavian, Michel J. P. Gingras, and Benjamin Canals, "Dynamically Induced Frustration as a Route to a Quantum Spin Ice State in $\mathrm{Tb}_{2} \mathrm{Ti}_{2} \mathrm{O}_{7}$ via Virtual Crystal Field Excitations and Quantum Many-Body Effects," Phys. Rev. Lett. 98, 157204 (2007).

[26] Shigeki Onoda and Yoichi Tanaka, "Quantum melting of spin ice: Emergent cooperative quadrupole and chirality," Phys. Rev. Lett. 105, 047201 (2010).

[27] R. Applegate, N. R. Hayre, R. R. P. Singh, T. Lin, A. G. R. Day, and M. J. P. Gingras, "Vindication of $\mathrm{Yb}_{2} \mathrm{Ti}_{2} \mathrm{O}_{7}$ as a Model Exchange Quantum Spin Ice," Phys. Rev. Lett. 109, 097205 (2012).

[28] Lieh-Jeng Chang, Shigeki Onoda, Yixi Su, Ying-Jer Kao, Ku-Ding Tsuei, Yukio Yasui, Kazuhisa Kakurai, and Martin Richard Lees, "Higgs transition from a magnetic Coulomb liquid to a ferromagnet in $\mathrm{Yb}_{2} \mathrm{Ti}_{2} \mathrm{O}_{7}$," Nature Communications 3, 992 (2012).

[29] Yi-Ping Huang, Gang Chen, and Michael Hermele, "Quantum Spin Ices and Topological Phases from Dipolar-Octupolar Doublets on the Pyrochlore Lattice," Phys. Rev. Lett. 112, 167203 (2014).

[30] SungBin Lee, Shigeki Onoda, and Leon Balents, "Generic quantum spin ice," Phys. Rev. B 86, 104412 (2012).

[31] Z. L. Dun, J. Trinh, K. Li, M. Lee, K. W. Chen, R. Baumbach, Y. F. Hu, Y. X. Wang, E. S. Choi, B. S. Shastry, A. P. Ramirez, and H. D. Zhou, "Magnetic Ground States of the Rare-Earth Tripod Kagome Lattice $\mathrm{Mg}_{2} \mathrm{RE}_{3} \mathrm{Sb}_{3} \mathrm{O}_{14}$ ( $\left.\mathrm{RE}=\mathrm{Gd}, \mathrm{Dy}, \mathrm{Er}\right)$," Phys. Rev. Lett. 116, 157201 (2016).

[32] Zhaofeng Ding, Yanxing Yang, Jian Zhang, Cheng Tan, Zihao Zhu, Gang Chen, and Lei Shu, "Possible gapless spin liquid in a rare-earth kagome lattice magnet $\mathrm{Tm}_{3} \mathrm{Sb}_{3} \mathrm{Zn}_{2} \mathrm{O}_{14}, "$ arXiv:1802.00968 (2018).

[33] Yuesheng Li, Haijun Liao, Zhen Zhang, Shiyan Li, Feng Jin, Langsheng Ling, Lei Zhang, Youming Zou, Li Pi,
Zhaorong Yang, Junfeng Wang, Zhonghua Wu, and Qingming Zhang, "Gapless quantum spin liquid ground state in the two-dimensional spin-1/2 triangular antiferromagnet $\mathrm{YbMgGaO}_{4}$," Sci. Rep. 5, 16419 (2015).

[34] Yuesheng Li, Gang Chen, Wei Tong, Li Pi, Juanjuan Liu, Zhaorong Yang, Xiaoqun Wang, and Qingming Zhang, "Rare-Earth Triangular Lattice Spin Liquid: A Single-Crystal Study of $\mathrm{YbMgGaO}_{4}$," Phys. Rev. Lett. 115, 167203 (2015).

[35] Yuesheng Li, Devashibhai Adroja, Pabitra K. Biswas, Peter J. Baker, Qian Zhang, Juanjuan Liu, Alexander A. Tsirlin, Philipp Gegenwart, and Qingming Zhang, "Muon Spin Relaxation Evidence for the U(1) Quantum Spin-Liquid Ground State in the Triangular Antiferromagnet $\mathrm{YbMgGaO}_{4}$," Phys. Rev. Lett. 117, 097201 (2016).

[36] Yao Shen, Yao-Dong Li, Hongliang Wo, Yuesheng Li, Shoudong Shen, Bingying Pan, Qisi Wang, H. C. Walker, P. Steffens, M. Boehm, Yiqing Hao, D. L. Quintero-Castro, L. W. Harriger, M. D. Frontzek, Lijie Hao, Siqin Meng, Qingming Zhang, Gang Chen, and Jun Zhao, "Evidence for a spinon fermi surface in a triangular-lattice quantum-spin-liquid candidate," Nature 540, 559-562 (2016).

[37] Joseph A. M. Paddison, Marcus Daum, Zhiling Dun, Georg Ehlers, Yaohua Liu, Matthew B. Stone, Haidong Zhou, and Martin Mourigal, "Continuous excitations of the triangular-lattice quantum spin liquid ybmggao, Nature Physics 13, 117-122 (2017).

[38] Y. Xu, J. Zhang, Y. S. Li, Y. J. Yu, X. C. Hong, Q. M. Zhang, and S. Y. Li, "Absence of Magnetic Thermal Conductivity in the Quantum Spin-Liquid Candidate $\mathrm{YbMgGaO}_{4}$," Phys. Rev. Lett. 117, 267202 (2016).

[39] Xinshu Zhang, Fahad Mahmood, Marcus Daum, Zhiling Dun, Joseph A. M. Paddison, Nicholas J. Laurita, Tao Hong, Haidong Zhou, N. P. Armitage, and Martin Mourigal, "Hierarchy of Exchange Interactions in the Triangular-Lattice Spin Liquid $\mathrm{YbMgGaO}_{4}$," Phys. Rev. X 8, 031001 (2018).

[40] Yuesheng Li, Devashibhai Adroja, Robert I. Bewley, David Voneshen, Alexander A. Tsirlin, Philipp Gegenwart, and Qingming Zhang, "Crystalline ElectricField Randomness in the Triangular Lattice Spin-Liquid $\mathrm{YbMgGaO}_{4}$," Phys. Rev. Lett. 118, 107202 (2017).

[41] Yuesheng Li, Sebastian Bachus, Yoshifumi Tokiwa, Alexander A Tsirlin, and Philipp Gegenwart, "Absence of zero-point entropy in a triangular ising antiferromagnet," arXiv preprint arXiv:1804.00696 (2018).

[42] Yuesheng Li, Devashibhai Adroja, David Voneshen, Robert I. Bewley, Qingming Zhang, Alexander A. Tsirlin, and Philipp Gegenwart, "Nearest-neighbour resonating valence bonds in $\mathrm{YbMgGaO}_{4}$," Nature Communications 8, 15814 (2017).

[43] Zhen Ma, Jinghui Wang, Zhao-Yang Dong, Jun Zhang, Shichao Li, Shu-Han Zheng, Yunjie Yu, Wei Wang, Liqiang Che, Kejing Ran, Song Bao, Zhengwei Cai, P. ermk, A. Schneidewind, S. Yano, J. S Gardner, Xin Lu, Shun-Li Yu, Jun-Ming Liu, Shiyan Li, Jian-Xin Li, and Jinsheng Wen, "Spin-Glass Ground State in a Triangular-Lattice Compound $\mathrm{YbZnGaO}_{4}$," Phys. Rev. Lett. 120, 087201 (2018).

[44] Yao Shen, Yao-Dong Li, H. C. Walker, P Steffens, M. Boehm, Xiaowen Zhang, Shoudong Shen, Hongliang Wo, Gang Chen, and Jun Zhao, "Fractionalized ex- 
citations in the partially magnetized spin liquid candidate $\mathrm{YbMgGaO}_{4}$," arXiv:1708.06655, Nature Communications.

[45] Yao-Dong Li, Xiaoqun Wang, and Gang Chen, "Anisotropic spin model of strong spinorbit-coupled triangular antiferromagnets," Phys. Rev. B 94, 035107 (2016).

[46] Yao-Dong Li, Xiaoqun Wang, and Gang Chen, "Hidden multipolar orders of dipole-octupole doublets on a triangular lattice," Phys. Rev. B 94, 201114 (2016).

[47] Changle Liu, Yao-Dong Li, and Gang Chen, "Selective measurements of intertwined multipolar orders: Non-kramers doublets on a triangular lattice," Phys. Rev. B 98, 045119 (2018).

[48] S. H. Curnoe, "Structural distortion and the spin liquid state in $\mathrm{Tb}_{2} \mathrm{Ti}_{2} \mathrm{O}_{7}$," Phys. Rev. B 78, 094418 (2008).

[49] Shigeki Onoda and Yoichi Tanaka, "Quantum fluctuations in the effective pseudospin- $\frac{1}{2}$ model for magnetic pyrochlore oxides," Phys. Rev. B 83, 094411 (2011).

[50] Yao-Dong Li, Yuan-Ming Lu, and Gang Chen, "Spinon Fermi surface $U(1)$ spin liquid in the spin-orbitcoupled triangular-lattice Mott insulator $\mathrm{YbMgGaO}_{4}$," Phys. Rev. B 96, 054445 (2017).

[51] Edward Parker and Leon Balents, "Finite-temperature behavior of a classical spin-orbit-coupled model for $\mathrm{YbMgGaO}_{4}$ with and without bond disorder,"
Phys. Rev. B 97, 184413 (2018).

[52] Yao-Dong Li, Yao Shen, Yuesheng Li, Jun Zhao, and Gang Chen, "Effect of spin-orbit coupling on the effective-spin correlation in $\mathrm{YbMgGaO}_{4}$," Phys. Rev. B 97, 125105 (2018).

[53] Yao-Dong Li and Gang Chen, "Detecting spin fractionalization in a spinon Fermi surface spin liquid," Phys. Rev. B 96, 075105 (2017).

[54] Zhenyue Zhu, P. A. Maksimov, Steven R. White, and A. L. Chernyshev, "Disorder-Induced Mimicry of a Spin Liquid in $\mathrm{YbMgGaO}_{4}$," Phys. Rev. Lett. 119, 157201 (2017).

[55] Zhenyue Zhu, P. A. Maksimov, Steven R. White, and A. L. Chernyshev, "Topography of Spin Liquids on a Triangular Lattice," Phys. Rev. Lett. 120, 207203 (2018).

[56] Itamar Kimchi, Adam Nahum, and T. Senthil, "Valence Bonds in Random Quantum Magnets: Theory and Application to $\mathrm{YbMgGaO}_{4}$," Phys. Rev. X 8, 031028 (2018).

[57] T. Senthil, "Theory of a continuous mott transition in two dimensions," Phys. Rev. B 78, 045109 (2008).

[58] M. Baenitz, Ph. Schlender, J. Sichelschmidt, Y. A. Onykiienko, Z. Zangeneh, K. M. Ranjith, R. Sarkar, L. Hozoi, H. C. Walker, J.-C. Orain, H. Yasuoka, J. van den Brink, H. H. Klauss, D. S. Inosov, and Doert Th., "NaYbS 2 - a new planar spin-1/2 triangular-lattice magnet and putative spin liquid," arXiv:1809.01947. 